\title{
IPEX syndrome: an easily-missed diagnosis of a life threatening condition
}

\author{
Abeer Alassaf, Rasha Odeh \\ Pediatric Endocrinology Division, The University of Jordan, 11942, P.O.Box 13046, Queen Rania Street, Amman, Jordan. \\ E-mail:drabeerassaf@hotmail.com \\ Received: 9th November 2017, Revised: 22nd January 2018, Accepted: 3rd February 2018
}

SUMMARY: Alassaf A, Odeh R. IPEX syndrome: an easily-missed diagnosis of a life threatening condition. Turk J Pediatr 2019; 61: 424-427.

We are reporting a case of neonatal diabetes mellitus, eczema and chronic diarrhea, suspected clinically to have Immunodysregulation Polyendocrinopathy Enteropathy X-linked (IPEX) syndrome and the diagnosis was confirmed by genetic testing. IPEX syndrome is a rare inherited X-linked recessive condition, causing life-threatening systemic autoimmune disorder which is characterized by immune dysregulation, polyendocrinopathy and enteropathy. The child who presented to our clinic at 11 months of age, had diabetic ketoacidosis at the age of 3 weeks, and was managed at that time and then was started on multiple dose insulin injection regimen, also he had a history of recurrent episodes of eczematous dermatitis and chronic diarrhea that started since early infancy. The rarity of this condition, may had led to delayed diagnosis and hence delayed definitive treatment - namely bone marrow transplant, which has variable outcomes. Immunosuppressive treatment can be used, if bone marrow transplant is not feasible.

Our aim is to increase the awareness of physicians to this rare syndrome, which usually presents as neonatal diabetes mellitus, eczematous dermatitis and chronic diarrhea. The earlier the diagnosis of this condition, the better the outcome.

Key words: IPEX syndrome, neonatal, diabetes mellitus, autoimmune disease.

The IPEX syndrome (Immunodysregulation Polyendocrinopathy Enteropathy X-linked syndrome) is a rare primary immunodeficiency disease. ${ }^{1}$ It is usually fatal within the first two years of life. ${ }^{2}$ Despite its complications, bone marrow transplantation remains the definitive mode of treatment. ${ }^{3}$ The earlier the diagnosis of this fatal condition, coupled with the initiation of treatment, the better the outcome with less morbidity and mortality. We report a child with IPEX syndrome diagnosed at the age of 11 months, although he developed neonatal diabetes mellitus at the age of 3 weeks as the first manifestation.

\section{Case Report}

A male infant presented to our center at the age of 11 months with the history of type 1 diabetes mellitus diagnosed at the age of 3 weeks. He was treated with a regimen of multiple daily insulin injections using detemir and short-acting insulin. He was born at term, with birth weight of $3.3 \mathrm{~kg}$. He is the youngest child to his first cousin parents, with healthy older brother and sister and absent family history of a similar condition. He had recurrent episodes of severe diarrhea and eczematous rash mainly on scalp and trunk. His physical examination revealed weight and length below the $5^{\text {th }}$ percentile, alopecia (Fig. 1), patches of eczematous rash on scalp and trunk (Fig. 2), and no obvious dysmorphic features.

The clinical diagnosis of IPEX syndrome was confirmed by genetic testing at the molecular genetics laboratory at the University of Exeter. Sanger sequence analysis of FOXP3 (Fig. 3) 


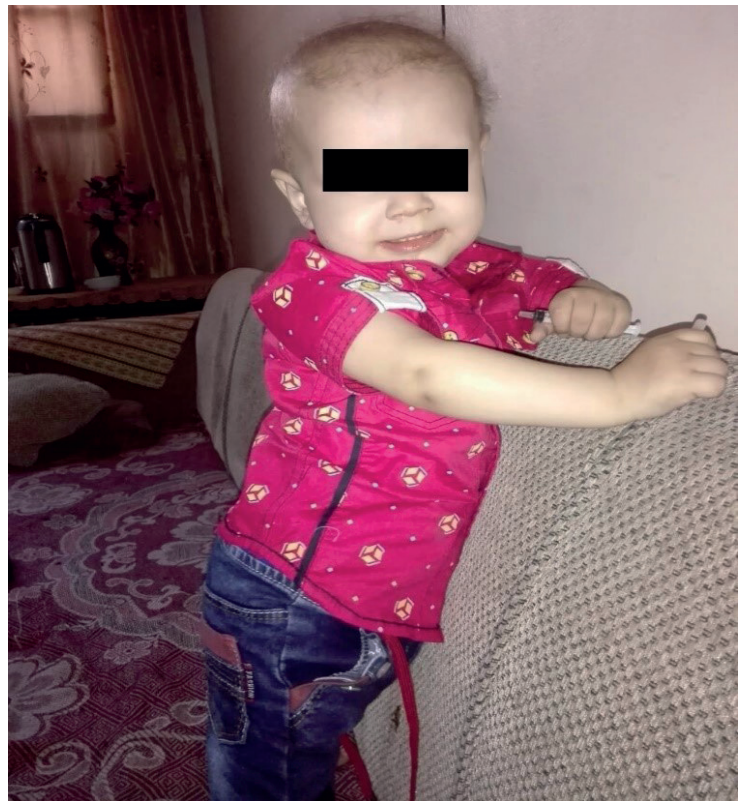

Fig. 1. Alopecia in a patient with IPEX syndrome.

identified a hemizygous pathogenic variant (c.1150G >A; p.Ala384Thr). This variant was not detected in the mother and thus is likely to be de novo.

\section{Discussion}

The presented patient was suspected to have IPEX syndrome at the age of 11 months, when he was seen in the author's Pediatric Endocrinology clinic despite the earlier development of neonatal diabetes mellitus. The clinical manifestations of IPEX syndrome, first described in $1982^{1}$, usually start during the first few months of life. Our patient was diagnosed with neonatal diabetes mellitus at the age of 3 weeks, presenting with diabetic ketoacidosis and a HbAlc of $12.5 \%$. He was started on detemir and short-acting insulin with moderate glycemic control. IPEXrelated diabetes mellitus is found in $4 \%$ of persistent neonatal diabetes mellitus and is unique because of its autoimmune nature. ${ }^{4}$ Our patient started to have recurrent episodes of eczematous rash mostly on the scalp and trunk (Fig. 2). A large number of IPEX patients, have dermatitis with a frequency of $65 \%{ }^{5}$. Since the age of one month, our patient started to have recurrent episodes of severe diarrhea, which required oral rehydration solution. Autoimmune enteropathy is

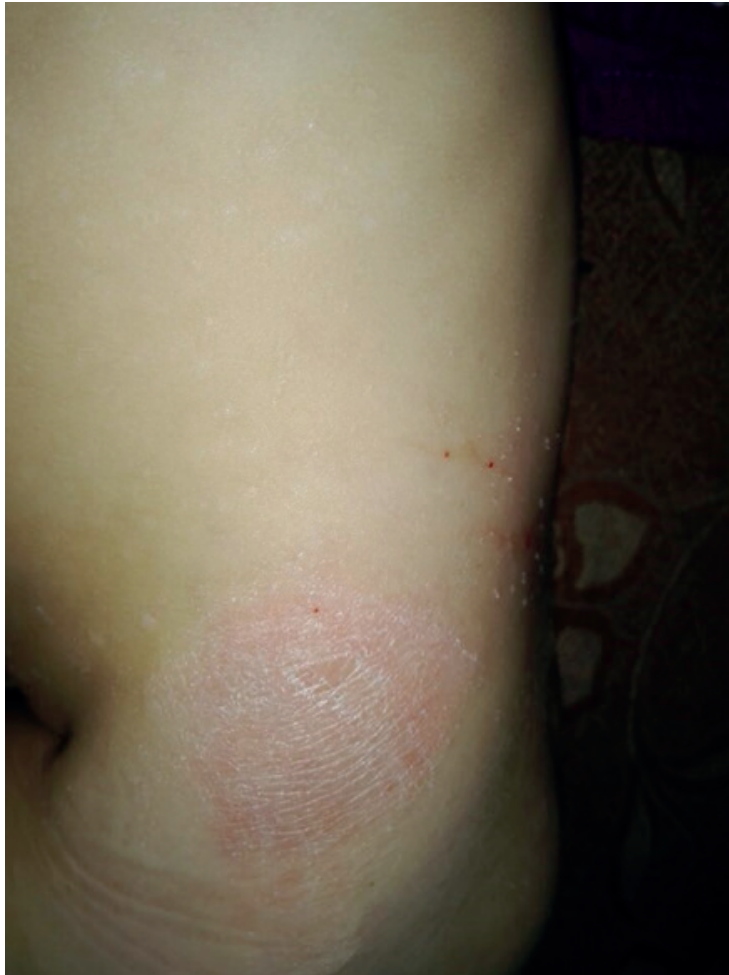

Fig. 2. Eczematous rash on the left loin.

universally present in patients with IPEX syndrome (100\%)..$^{5}$ Growth parameters of our patient were below the $5^{\text {th }}$ percentile. Patients with IPEX syndrome, usually have growth retardation due to chronic inflammation with high serum cytokines, autoimmune enteropathy and diabetes mellitus. ${ }^{6}$

Other manifestations of IPEX syndrome may also be present, including alopecia which was found in our patient (Fig. 1), in addition to thyroiditis $(30 \%)$, recurrent infections $(20 \%)$ and less common additional autoimmune conditions like autoimmune cytopenias, nephritis, pneumonitis, hepatitis, arthritis, myositis and vasculitis. ${ }^{5,7}$ Manifestations of IPEX syndrome usually appear sequentially, and the affected organ spectrum varies from one patient to another. ${ }^{5}$ Without treatment, IPEX syndrome is usually fatal within the first 2 years of life due to life-threatening infections, severe watery diarrhea and diabetic ketoacidosis. ${ }^{9}$ Milder phenotypes of IPEX syndrome have been reported in a number of patients, who can live longer. Enteropathy was present in all of them, although diabetes was frequently absent. ${ }^{4,10,11}$ 

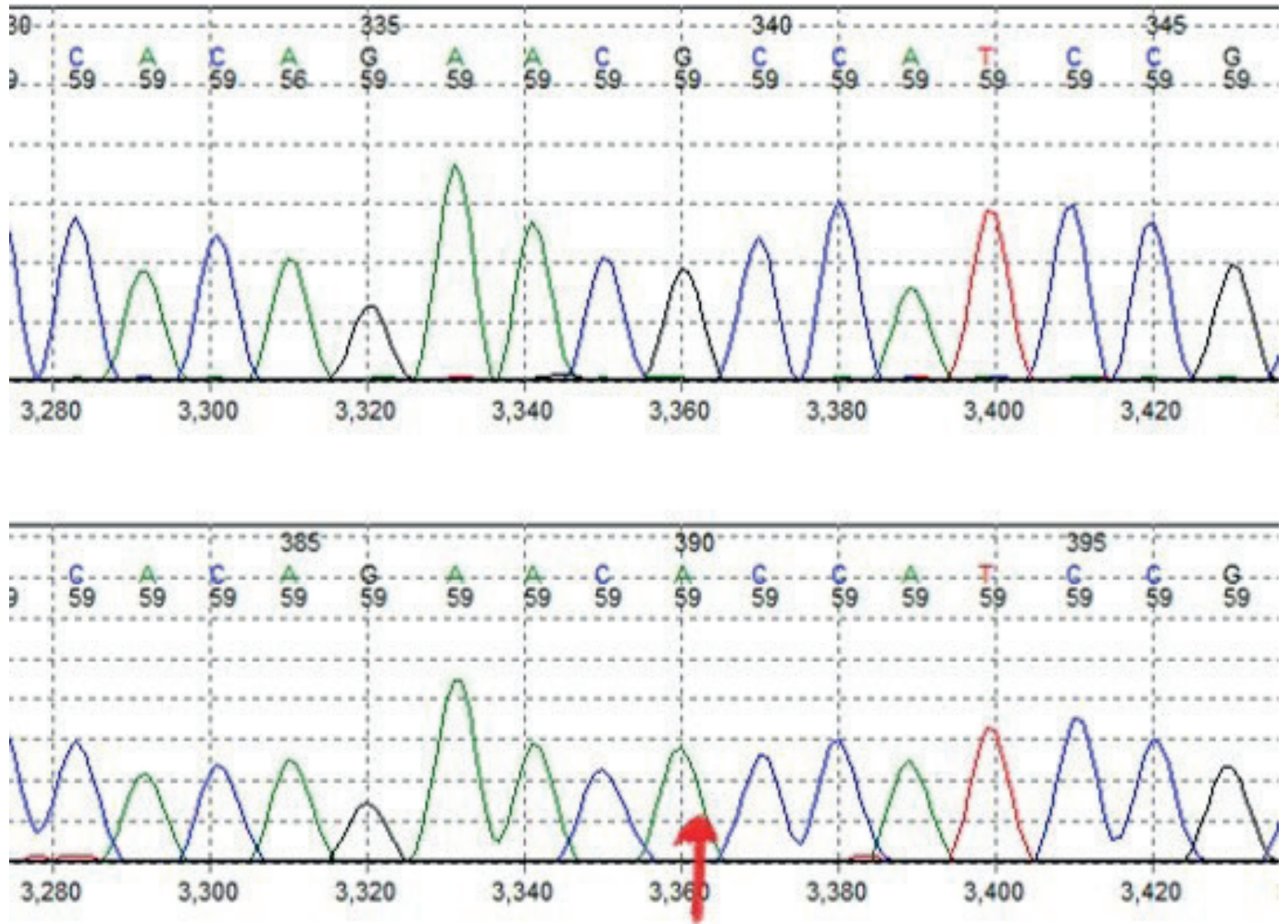

Fig. 3. A section of the Sanger sequence of FOXP3 with the arrow pointing to the nucleotide change.

We suspected our patient to have IPEX syndrome based on the characteristic clinical findings, mainly neonatal diabetes mellitus, eczematous rash and autoimmune enteropathy in addition to alopecia and failure to thrive. Genetic testing revealed a mutation in FOXP3 (c.1150G >A; p.Ala384Thr) thus confirming the diagnosis. FOXP3 maps to chromosome Xp11.23-Xq13.3 and encodes forkhead box protein 3 , required for the generation and functioning of $\mathrm{CD} 4(+) \mathrm{CD} 25(+)$ regulatory $\mathrm{T}$ lymphocytes, the potent suppressors of T-cell activation and proliferation. ${ }^{12,13}$ Deficiency of these cells results in an uncontrolled autoimmune activity. ${ }^{14}$ More than 60 mutations of FOXP3 have been detected so far. ${ }^{15}$ The mutation found in our patient has been described in an IPEX patient, it changes an important amino acid in the DNA binding domain of the forhead box protein 3 and is not found in an ethnically diverse control population. ${ }^{16}$

Supportive treatment of IPEX syndrome consists of parenteral nutrition, blood transfusions, and management of the diabetes mellitus. There is a paucity of data on clinical trials for definitive treatment of IPEX syndrome due to its rarity. Immunosuppressive therapy and bone marrow transplantation have shown some efficacy. Various immunosuppressive medications have been used including highdose steroids, cyclosporine A, tacrolimus, methotrexate, infliximab, rituximab and sirolimus. ${ }^{17,18}$ Allogenic bone marrow transplantation reduces symptoms of eczema, enteropathy and diabetes, although patients may experience complications such as infections and lymphoproliferative hemophagocytic syndrome. ${ }^{3}$ Our patient was referred to a specialized center for bone marrow transplant, immediately after diagnosis was confirmed by genetic testing, and we'll be following for updates on his clinical condition and response to treatment. Early treatment of IPEX syndrome with bone marrow transplant limits the autoimmune damage to endocrine organs and reduces the risk of infections. ${ }^{19}$

To our knowledge, this is the first IPEX 
syndrome patient reported from Jordan, and probably the Middle East. Our patient developed the classical manifestations of neonatal diabetes mellitus, followed by recurrent severe diarrhea and eczema, but due to the rarity of the condition, the diagnosis was delayed till the age of 11 months. In conclusion, physicians should be aware of this extremely rare condition, which could be easily identified by the constellation of its characteristic features. Earlier diagnosis leads to earlier intervention with better outcome.

\section{Acknowledgement}

The genetic testing was performed by the University of Exeter Medical School with funding from the Wellcome Trust. Authors are also grateful to professor Hatem El-Shanti for revising the final manuscript.

\section{REFERENCES}

1. Powell BR, Buist NR, Stenzel P. An X-linked syndrome of diarrhea, polyendocrinopathy, and fatal infection in infancy. J Pediatr 1982; 100: 731-737.

2. Bennett CL, Christie J, Ramsdell F, et al. The immune dysregulation, polyendocrinopathy, enteropathy, $\mathrm{X}$-linked syndrome (IPEX) is caused by mutations of FOXP3. Nat Genet 2001; 27: 20-21.

3. Baud O, Goulet O, Canioni D, et al. Treatment of the immune dysregulation, polyendoccrinopathy, enteropathy, $\mathrm{X}$ linked syndrome (IPEX) by allogeneic bone marrow transplantation. N Engl J Med 2001; 344: 1758-1762.

4. Rubio-Cabezas O, Minton JAL, Caswell R, et al. Clinical heterogeneity in patients with FOXP3 mutations presenting with permanent neonatal diabetes. Diabetes Care 2009; 32: 111-116.

5. Moraes-Vasconcelos D, Costa-Carvalho BT, Torgerson TR, Ochs HD. Primary immune deficiency disorders presenting as autoimmune diseases: IPEX and APECED. J Clin Immunol 2008; 28(Suppl 1): S11-S19.

6. Torgerson TR. Regulatory $\mathrm{T}$ cells in human autoimmune diseases. Springer Semin Immunopathol 2006; 28: 63-76.

7. Wildin RS, Smyk-Pearson S, Filipovich AH. Clinical and molecular features of the immunodysregulation, polyendocrinopathy, enteropathy, $\mathrm{X}$ linked (IPEX) syndrome. J Med Genet 2002; 39: 537-545.
8. Savova R, Arshinkova M, Houghton J, et al. Clinical case of immune dysregulation, polyendocrinopaty, enteropathy, X-linked (IPEX) syndrome with severe immune deficiency and late onset of endocrinopathy and enteropathy. Case Rep Med 2014; 2014: 564926.

9. Bennett CL, Ochs HD. IPEX is a unique X-linked syndrome characterized by immune dysfunction, polyendocrinopathy, enteropathy, and a variety of autoimmune phenomena. Curr Opin Pediatr 2001; 13: $533-538$.

10. De Benedetti $F$, Insalaco A, Diamanti $A$, et al. Mechanistic associations of a mild phenotype of immunodysregulation, polyendocrinopathy, enteropathy, x-linked syndrome. Clin Gastroenterol Hepatol 2006; 4: 653-659.

11. Bacchetta R, Passerini L, Gambineri E, et al. Defective regulatory and effector $\mathrm{T}$ cell functions in patients with FOXP3 mutations. J Clin Invest 2006; 116: 1713-1722.

12. Gavin MA, Rasmussen JP, Fontenot JD, et al Foxp3-dependent programmeo of regulatory T-cell differentiation. Nature 2007; 445: 771-775.

13. Wan YY, Flavell RA. Regulatory T-cell functions are subverted and converted owing to attenuated Foxp3 expression. Nature 2007; 445: 766-770.

14. Valencia X, Lipsky PE. CD4+CD25+FoxP3+ regulatory $\mathrm{T}$ cells in autoimmune diseases. Nat Clin Pract Rheumatol 2007; 3: 619-626.

15. Bin Dhuban K, Piccirillo CA. The immunological and genetic basis of immune dysregulation, polyendocrinopathy, enteropathy, X-linked syndrome. Curr Opin Allergy Clin Immunol 2015; 15: 525-532.

16. Wildin RS, Ramsdell F, Peake J, et al. X-linked neonatal diabetes mellitus, enteropathy and endocrinopathy syndrome is the human equivalent of mouse scurfy. Nat Genet 2001; 27: 18-20.

17. Kobayashi I, Nakanishi M, Okano M, Sakiyama Y, Matsumoto S. Combination therapy with tacrolimus and betamethasone for a patient with X-linked autoimmune enteropathy. Eur J Pediatr 1995; 154: 594595.

18. Kobayashi I, Kawamura N, Okano M. A longterm survivor with the immune dysregulation, polyendocrinopathy, enteropathy, X-linked syndrome. N Engl J Med 2001; 345: 999-1000.

19. Rao A, Kamani N, Filipovich A, et al. Successful bone marrow transplantation for IPEX syndrome after reduced-intensity conditioning, Blood 2007; 109: 383-385. 